\title{
FUZZY RELIABILITY ANALYSIS OF COMPOUND SYSTEM BASED ON WEIBULL DISTRIBUTION
}

\author{
Yunfei Guo
}

Department of Mathematics, Yanbian University, Yanji, 133002, P. R. China

\begin{abstract}
Data in the real world can not be recorded or collected precisely due to human errors, machine errors, or some other unexpected situations. And reliability analysis is an important research topic in engineering. The data, however, can not be recorded precisely sometimes as described above. Then some researchers pay attention to the fuzzy reliability. In this paper, we are going to analyze the fuzzy reliability of compound system, which obeys the Weibull distribution. For the compound system composed of multiple components, $C_{1}, C_{2}, \ldots, C_{n}$, especially when $\eta=1$, we obtain the corresponding fuzzy reliability function. Besides, values of reliability of compound system with various $n$ and $m$ have been calculated. Finally, necessary reliability analysis is also provided in the paper.
\end{abstract}

Keywords: compound system, fuzzy reliability, Weibull distribution.

${ }^{*}$ Corresponding author.

E-mail address: guoyunfei0413@sina.com (Yunfei Guo).

Copyright (c) 2015 Scientific Advances Publishers 2010 Mathematics Subject Classification: 62N05.

Submitted by Yewang Chen.

Received August 17, 2015; Revised August 29, 2015 


\section{Introduction}

Zadeh [1] gradually established operation, transformation rule and carried out the relevant theoretical research on the "fuzzy sets" based on the precise mathematical set theory. Fuzzy set theory was first introduced as a useful tool to complement conventional reliability theories in [2]. Since then, there have been a number of approaches where the technology of fuzzy sets was used to evaluate system reliability. For example, in Bing et al. [3], a fuzzy linear regression method is combined with a finite element method to evaluate the reliability of mechanical structures. In this approach, a membership function of a triangular fuzzy number is used to express the structure stress. In order to overcome the limitation of the traditional failure mode, effects and criticality analysis, a fuzzy rule-based approach has been implemented in [4, 5, 6]. Furthermore, Zio et al. [7] developed a fuzzy expert system for human reliability analysis to elicitate factors influencing conditional human error for two dependence successive operator actions in a nuclear power plant accident. In Karimi and Hüllermeier [8], fuzzy set theory has been used to complement probability theory to assess the risk of natural disaster when statistical data and/or physical knowledge are insufficient for probabilistic analysis. Meanwhile, Ding and Lisnianski [9] developed a fuzzy universal generating function in which fuzzy numbers used to represent the state

probability and fuzzy composition operators were introduced to assess the reliability of a multi-state system. Moreover, Pandey and Tyagi [10] proposed a profust reliability to evaluate degradable systems and a fuzzy numbers-based method to assess system failure rate parameters. $\mathrm{Li}$ and Gao [11] analyzed and deduced fuzzy reliability of paralleling system in the paper. Zhang and Chen [12] obtained the expression about reliability index by building and solving the model. 


\section{Pre- Knowledge}

Compound system in this paper is a system whose graphic is in the following:

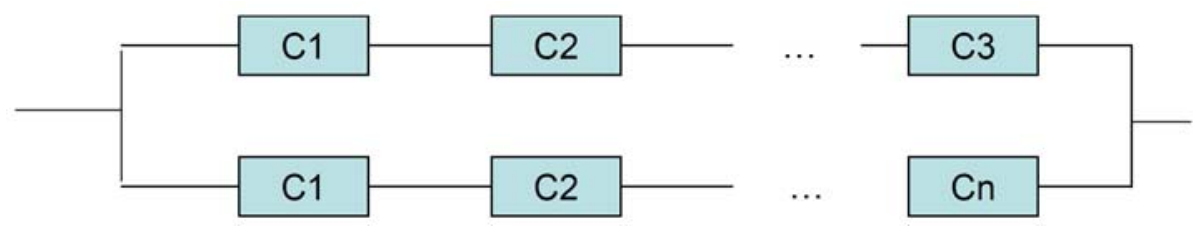

Figure 1. The compound system.

Definition 2.1 ([11]). Li and Gao defined the following symbols:

$C$ : state that system works normally.

$C_{j}$ : state that the $j$-th component works normally.

$\underset{\sim}{A} i$ : fuzzy state set.

They also obtained the following formulas:

$$
\left\{\begin{array}{l}
\bar{C}=\bigcap_{j=1}^{n} \bar{C}_{j}, \quad P(\bar{C})=P\left(\bigcap_{j=1}^{n} \bar{C}_{j}\right), \\
C=1-\bigcap_{j=1}^{n} \bar{C}_{j}
\end{array}\right.
$$

If the failure of the parts were independent each other, then

$$
\begin{cases}\text { The system of common failure rate } & P(\bar{C})=\prod_{j=1}^{n} \bar{C}_{j}, \\ \text { The system of fuzzy failure rate } & P\left(C \Delta \sim_{\sim}^{A}\right)=P\left({\underset{\sim}{\sim}}_{i} \mid C\right) P(C), \\ \text { The part of fuzzy failure rate } & P\left(C_{j} \Delta \underset{\sim}{A_{i}}\right)=P\left(\underset{\sim}{\left.A_{i} \mid C_{j}\right) P\left(C_{j}\right) .}\right.\end{cases}
$$


Definition 2.2 ([11]). According to the general reliability theory and literature, we can know that

$$
\begin{aligned}
& P(C)=R_{s}, P\left(C_{j}\right)=R_{j}, \\
& P\left(C \Delta \underset{\sim}{A_{i}}\right)=\underset{\sim}{R}, P\left(C_{j} \Delta \underset{\sim}{A} i\right)=\underset{\sim}{R}, \\
& P\left(A_{i} \mid C\right)=\mu_{\sim}^{A_{i}}\left(R_{s}\right), P\left(\underset{\sim}{A_{i}} \mid C_{j}\right)=\mu_{\sim}^{A_{i}}\left(R_{j}\right) .
\end{aligned}
$$

Lemma 2.1 ([13]). The failure distribution of paralleling system obeys Weibull distribution Wei( $m, \eta)$. Two parameters of Weibull distribution are $\eta, m$. Among them, $\eta$ is referred to the characteristics of life, and $m$ is referred to the shape parameter, where $\eta>0, m>0$. Wei $(m, \eta)$ 's information is the following:

Distribution function:

$$
F(t)=1-\exp \left\{-\left(\frac{t}{\eta}\right)^{m}\right\}, \quad t>0 .
$$

Density function:

$$
f(t)=[F(t)]^{\prime}=\frac{m}{\eta}\left(\frac{t}{\eta}\right)^{m-1} \cdot \exp \left\{-\left(\frac{t}{\eta}\right)^{m}\right\}, \quad t>0 .
$$

Failure function:

$$
\lambda(t)=\frac{f(t)}{1-F(t)}=\frac{m}{\eta}\left(\frac{t}{\eta}\right)^{m-1}, \quad t>0 .
$$

Lemma 2.2. According to Equations (2.4), (2.5), (2.6) above, we can obtain that

The reliability function:

$$
R(t)=1-F(t)=\exp \left\{-\left(\frac{t}{\eta}\right)^{m}\right\}, \quad t>0 .
$$




\section{Fuzzy Reliability Analysis in Early Failure Mode}

According to the Lemma 2.1, when $m<1$, it is in early failure mode.

For a component paralleling system composed of $n$ parts, $C_{1}, C_{2}, \ldots, C_{n}$. The analysis of fuzzy reliability is in the following.

Assume that each part's failure is independent each other, and $\eta=1, m_{j}=m, j=1, \cdots, n$, and it obeys $\operatorname{Wei}(m, \eta)$, then fuzzy reliability function of compound system can be obtained.

Theorem 3.1. The fuzzy reliability function of compound system:

$$
\underset{\sim}{R}(t)=\mu_{\sim}^{A_{i}}\left(R_{s}\right)\left(2-e^{-2}\right) \exp \left(-n t^{m}\right), \quad t>0,
$$

when $\eta=1$ and $m_{j}=m, j=1, \cdots, n$.

Proof. First of all, when $\eta=1$

$$
R_{j}(t)=1-F(t)=\exp \left(-t^{m_{j}}\right), \quad j=1, \cdots, n, \quad t>0 .
$$

From reference [14], we can obtain that

$$
\begin{aligned}
R_{s}(t) & =1-\left[1-\prod_{j=1}^{n} R_{j}\right]^{2}=2 \prod_{j=1}^{n} R_{j}-\left(\prod_{j=1}^{n} R_{j}\right)^{2} \\
& =2 \prod_{j=1}^{n} \exp \left(-t^{m_{j}}\right)-\left(\prod_{j=1}^{n} \exp \left(-t^{m_{j}}\right)\right)^{2} \\
& =2 \exp \left(-\sum_{j=1}^{n} t^{m_{j}}\right)-\exp \left(-2 \sum_{j=1}^{n} t^{m_{j}}\right) .
\end{aligned}
$$

When $m_{j}=m, j=1, \cdots, n$, we can obtain that

$$
\begin{aligned}
R_{s}(t) & =2 \exp \left(-n t^{m}\right)-\exp \left(-2 n t^{m}\right) \\
& =\left(2-e^{-2}\right) \exp \left(-n t^{m}\right)
\end{aligned}
$$


So $\underset{\sim}{R}(t)=\mu_{\sim}^{\mu_{i}}\left(R_{s}\right) R_{s}(t)=\mu_{\sim}^{A_{i}}\left(R_{s}\right)\left(2-e^{-2}\right) \exp \left(-n t^{m}\right)$.

From Theorem 3.1, we can see that fuzzy reliability function of compound system $\underset{\sim}{R}(t)$ is the function about time $t$, which includes two parameters $n$ and $m$. Different values of $\underset{\sim}{R}(t)$ with different $n$ and $m$ are provided in Table 1.

Table 1. Reliability of compound system with various $n$ and $m$

\begin{tabular}{|l|c|c|c|c|}
\hline$n-m$ & 0.1 & 0.3 & 0.5 & 0.7 \\
\hline 3 & 0.2809 & 0.4882 & 0.7221 & 0.9526 \\
\hline 4 & 0.1495 & 0.3123 & 0.5263 & 0.7615 \\
\hline 5 & 0.0795 & 0.1998 & 0.3836 & 0.6088 \\
\hline 6 & 0.0423 & 0.1278 & 0.2796 & 0.4867 \\
\hline
\end{tabular}

From Table 1, we can see the effects of two parameters $n$ and $m$. First of all, the reliability would increase when $m$ increases for each given $m$, this is because that the system is in the early failure mode, and the failure rate would decrease with the increasing $m$. On the other hand, the reliability would decrease when $n$ increases for each given $n$, reasons for this is that more components in the series system, lower reliability would system becomes. 
Figure 2 and Figure 3 provide more intuitive explanation.

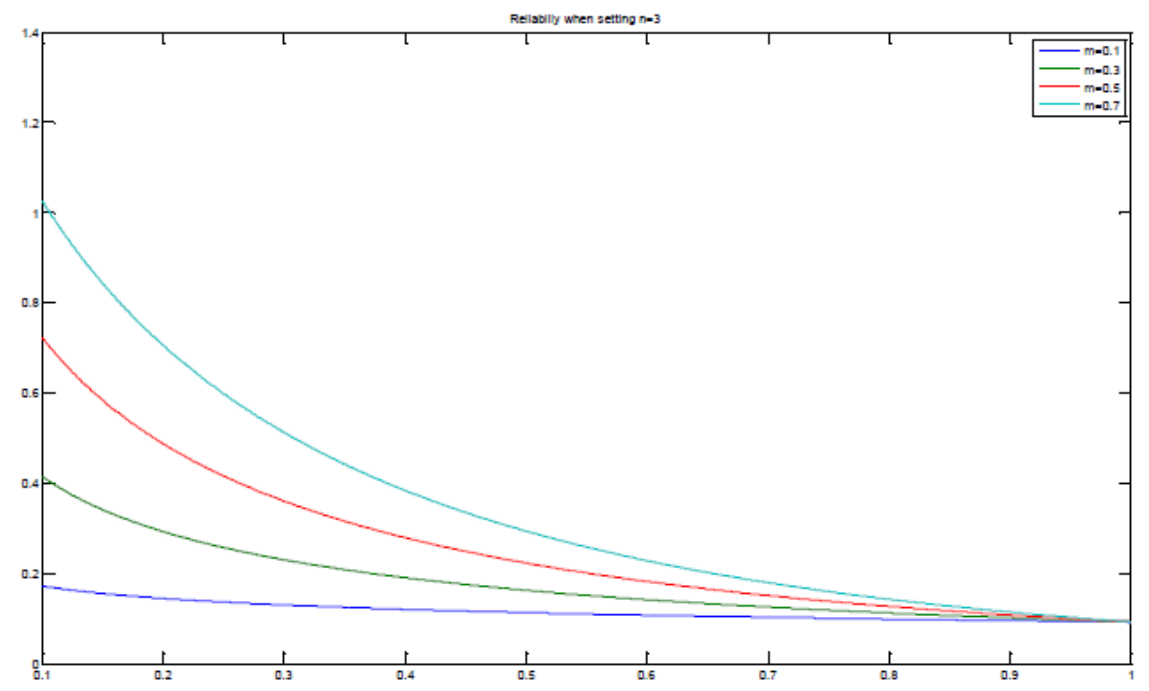

Figure 2. Reliability when setting $n=3$.

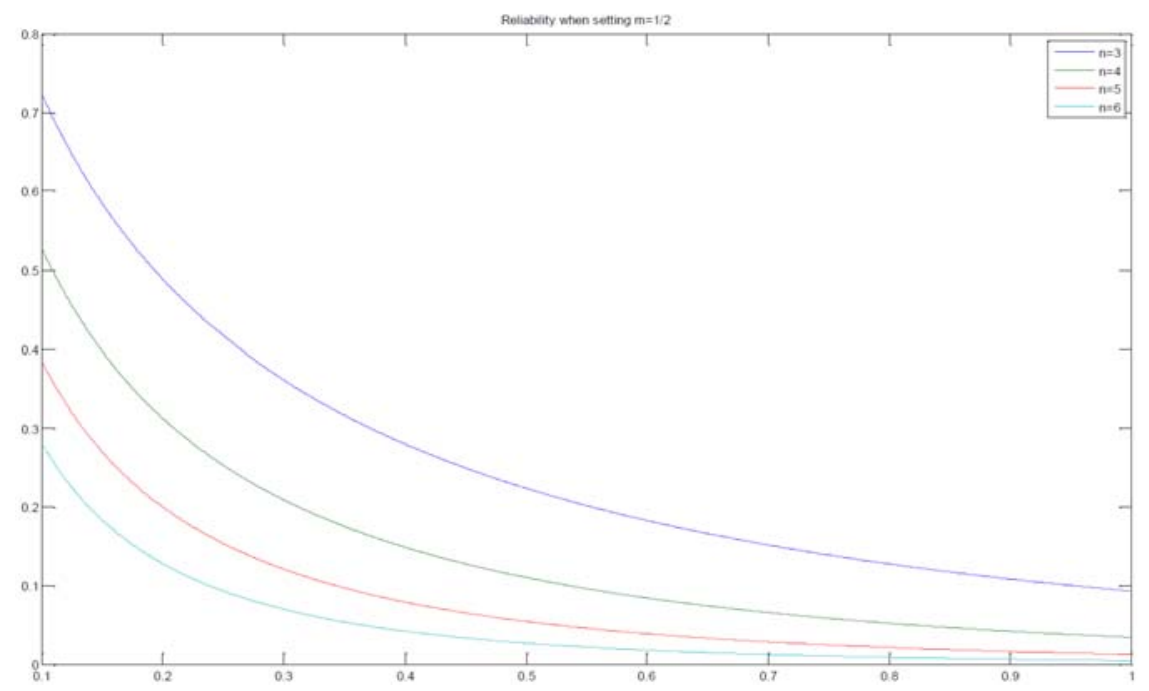

Figure 3. Reliability when setting $m=1 / 2$. 
Reliability analysis about compound system have been given above, as for fuzzy reliability, we would regard $\mu_{\sim}{ }_{i}\left(R_{s}\right)$ as the constant which is the mean $\mu_{\sim}{ }_{i}\left(R_{s}\right)_{m}$, and because $\underset{\sim s}{R}(t)=\mu_{\sim}^{\mu_{i}}\left(R_{s}\right) R_{s}(t)$, then reliability analysis are the same as fuzzy reliability analysis in this meaning.

\section{Conclusion}

In this paper, we analyze the fuzzy reliability of compound system, which obeys the Weibull distritution. For the compound system composed of multiple components, $C_{1}, C_{2}, \ldots, C_{n}$, especially when $\eta=1$, we obtain the corresponding fuzzy reliability function. Finally, necessary reliability analysis is also provided in the paper.

\section{References}

[1] L. A. Zadeh, Fuzzy sets, Information and Control 8(3) (1965), 338-353.

[2] T. Onisawa, Fuzzy theory in reliability analysis, Fuzzy Sets Syst. 30(3) (1989), 361-363.

[3] L. Bing, Z. Meilin and X. Kai, A practical engineering method for fuzzy reliability analysis of mechanical structures, Reliab. Eng. Syst. Saf. 67(3) (2000), 311-315.

[4] J. B. Bowles and C. E. Pelaez, Fuzzy logic prioritization of failures in a system failure mode, effects and criticality analysis, Reliab. Eng. Syst. Saf. 50(2) (2005), 203-213.

[5] H. Gargama and S. K. Chaturvedi, Criticality assessment models for failure mode effects and criticality analysis using fuzzy logic, IEEE Trans. Reliab. 60(1) (2011), 102-110.

[6] K. Xu, L. C. Tang, M. Xie, S. L. Ho and M. L. Zhu, Fuzzy assessment of FMEA for engine systems, Reliab. Eng. Syst. Saf. 75(1) (2002), 17-29.

[7] E. Zio, P. Baraldi, M. Librizzi, L. Podofillini and V. N. Dang, A fuzzy set-based approach for modeling dependence among human errors, Fuzzy Sets Syst. 160(13) (2009), 1947-1964.

[8] I. Karimi and E. Hüllermeier, Risk assessment system of natural hazards: A new approach based on fuzzy probability, Fuzzy Sets Syst. 158(9) (2007), 987-999.

[9] Y. Ding and A. Lisnianski, Fuzzy universal generating functions for multi-state system reliability assessment, Fuzzy Sets Syst. 159(3) (2008), 307-324. 
[10] D. Pandey and S. K. Tyagi, Profust reliability of a gracefully degradable system, Fuzzy Sets Syst. 158(7) (2007), 794-803.

[11] Li Tingjie and Gao He, The fuzzy reliability of the serial system, Fuzzy Systems and Mathematics 3(1) (1989), 38-45.

[12] Zhang Minyue and Chen Yanli, Reliability analysis of different types of three components in parallel systems, Journal of Lanzhou University of Technology 37(2) (2011), 127-130.

[13] Mao Shisong, Tang Yincai and Wang Lingling, Reliable Statistics, Higher Education Press, 2008.

[14] Chen Dexin, Fuzzy Reliability of Compound Systems (I), System Engineering-Theory \& Practice, 1997. 